\title{
Research on the Inter-Cultural Communication of Yanzhao Traditional Sports Culture under the Background of the Winter Olympics
}

\author{
Huijian Wang1, Ying Huang1, Yi Yang1, Yalong Li2 ${ }^{*}$ \\ ${ }^{1}$ College of Foreign Language Education and International Business, Baoding University, Baoding, China \\ ${ }^{2}$ College of Physical Education, Baoding University, Baoding, China \\ Email: 80701401@qq.com, *suibianlong@126.com
}

How to cite this paper: Wang, H. J., Huang, Y., Yang, Y., \& Li, Y. L. (2021). Research on the Inter-Cultural Communication of Yanzhao Traditional Sports Culture under the Background of the Winter Olympics. Advances in Physical Education, 11, 261-267.

https://doi.org/10.4236/ape.2021.112021

Received: April 13, 2021

Accepted: May 16, 2021

Published: May 19, 2021

Copyright $\odot 2021$ by author(s) and Scientific Research Publishing Inc. This work is licensed under the Creative Commons Attribution International License (CC BY 4.0).

http://creativecommons.org/licenses/by/4.0/

\begin{abstract}
Yanzhao Traditional Sports is an intangible cultural heritage with distinctive national characteristics. Taking advantage of the opportunity of the 2022 Beijing-Zhangjiakou Winter Olympics, researching the inter-cultural communication of Yanzhao traditional sports and constructing an effective communication model will help grasp the initiative of the inter-cultural communication of Yanzhao traditional sports culture and shape the image of Hebei. In order to promote inter-cultural communication of Yanzhao traditional sports culture, it is advised to construct from improving the awareness of inter-cultural communication, selection of intercultural communication content, and broaden the channels of inter-cultural communication.
\end{abstract}

\section{Keywords}

Inter-Cultural Communication, Yanzhao Traditional Sports Culture, Winter Olympics

\section{Introduction}

Sports culture is an important part of the national spirit and the spirit of the times. It is a powerful spiritual force that inspires everyone to work hard and is the soul of the leap-forward development of sports. The traditional sports culture of Yanzhao is rich and has a deep humanistic foundation. It had absorbed the traditional Central Plains sports culture and grassland sports culture, and developed into a unique form of regional cultural expression with the changes of history.

The traditional sports culture of Yanzhao has the national characteristics 
and humanistic spirit of the region. The inter-cultural communication of Yanzhao traditional sports culture can show the whole world the vigorous and self-improving masculinity of Yanzhao traditional sports culture, and promote the advancement and development of the Chinese national sports. This paper aims to explore the ways to promote the intercultural communication of inter-cultural communication of Yanzhao traditional sports culture to make it known by foreigners and thus arouse their interest in learning more about Hebei province.

This paper is divided into 4 parts. Part 1 is the introduction. It includes the background, purpose, significance sand structure of the research. Part 2 is mainly about the opportunities and challenges that 2022 Beijing-Zhangjiakou Winter Olympic Games bring to inter-cultural communication of Yanzhao traditional sports culture. Part 3 is the core of the research. It is about the strategies that can help promote the inter-cultural communication of Yanzhao traditional sports culture, including enhancing the awareness of inter-cultural communication, delicate selection of intercultural content and broaden the channels of intercultural communication. Part 4 is the conclusion. It is the result and significance of the research.

\section{Opportunities and Challenges Brought by the Winter Olympics}

Preparing for the 2022 Winter Olympics is a rare historical opportunity to promote the inter-cultural communication of Yanzhao traditional sports. With the approach of the Winter Olympics, Hebei Province pays attention to infiltrating the awareness of local cultural characteristics and the concept of local cultural protection into Yanzhao Traditional Sports Culture Innovation Project. In the process of developing sports, it also more consciously enhances people's awareness fusion of local traditional sports culture and modern sports culture. At present, Hebei Province has expanded the inter-cultural communication of Yanzhao traditional sports culture to the construction of the sports culture of the 2022 Beijing-Zhangjiakou Winter Olympics and has initially constructed an inter-cultural communication system with certain local cultural characteristics, and integrates the characteristic forms of sports culture into the development of sports, which will play a very active role in realizing the inter-cultural communication of national culture.

Combined with the reality, the inter-cultural communication of Yanzhao traditional sports culture should adopt innovative concepts and channels. In the context of the Winter Olympics, the inter-cultural communication of Yanzhao traditional sports culture also faces a series of challenges.

Firstly, the form of Yanzhao traditional sports culture is facing the problem of "aging". The content of traditional sports culture in Yanzhao is mainly martial arts culture. Although martial arts culture is the essence of traditional sports culture in China, the modern way of inheriting traditional sports culture in Yanzhao is relatively single, which leads to the lack of innovation in the form of 
inter-cultural communication of Yanzhao traditional sports culture. Especially, it is difficult to attract the attention of young people.

Secondly, the biggest obstacle to inter-cultural communication of Yanzhao traditional sports culture is the lack of talents and the lack of communication personnel with professional knowledge. Language is the link of cross-cultural communication. Only by accurately translating traditional sports culture into foreign languages can it convey traditional sports and its profound cultural heritage, export the culture and values, achieve recognition and reduce misunderstandings, so that it can create a favorable international environment, for Chinese sports culture and achieve international communication and promotion (Tuo, 2017). Consulting the information, it is found that Hebei province lacks institutions and organizations to train relevant professionals, and there is a great need of intercultural communicators who understand both the language and the sports professional knowledge and sports culture. At the same time, due to the lack of professional guidance, the professional level of the current communicators is also uneven. It is mainly reflected in the fact that it is difficult for the learners of traditional sports culture to achieve communication ability. There is no shortage of inheritors of sports cultural heritage in Yanzhao, but the inheritors who can use a foreign language proficiently and spread the content of sports culture are far from satisfying the demand. These are major challenges for the inter-cultural communication and development of Yanzhao traditional sports culture.

\section{Intercultural Communication Strategies of Yanzhao Traditional Sports Culture}

\subsection{Enhancing the Awareness of Main Body of Inter-Cultural Communication of Yanzhao Traditional Sports Culture}

The development of inter-cultural communication of traditional sports culture in Yanzhao should not be limited to professional sports. Government agencies, universities, non-governmental organizations, and even event volunteers should become the main body of inter-cultural communication of sports culture, and all of them should cultivate awareness of inter-cultural communication of traditional sports culture and become the communicator of excellent sports culture.

The government should comprehensively plan the layout of inter-cultural communication work and carry out the work in a planned and step-by-step manner through the promulgation of relevant policies and regulations. Colleges and universities should train some professional talents (Jing, Gai, \& Xu, 2010), who are familiar with Yanzhao traditional sports culture as well as proficient in foreign languages, to become a main force of communication and transmit the latest information in a timely and effective way, which is conducive to expanding the scope of influence of inter-cultural communication and attracting more social groups and individuals to join. These measures will surely play a positive effect on promoting the inter-cultural communication of traditional sports culture. 


\subsection{Selection of Inter-Cultural Communication Content}

\subsubsection{Three-Dimensional Design}

The representative sports of Yanzhao traditional sports are: traditional martial arts such as Cangzhou martial arts, Handan Taichi; folk acrobatics such as Wuqiao acrobatics; traditional games such as Chengde Pearl ball, Laishui Kickball, Dragon Boat Racing, etc. These traditional sports cultures embody the concept of "Rule of morality"-Confucianism as the mainstream-combining body and soul, movement and static, keeping one's health and self-cultivation. They focus on the role and guidance of morality in sports, and focus on the cultivation of character and morality. They advocate the use of virtue to guide physical fitness and pay attention to the spiritual guidance and belief cultivation.

In the process of inter-cultural communication, the traditional sports culture of Yanzhao should be summarized and integrated, and elements that are popular and in line with the demands of the times should be explored to make it easier to be understood and accepted. Aiming at different levels of audiences, the three-dimensional design of the communication content of Yanzhao traditional sports culture is carried out to achieve the combination of point, line and surface. The audience can choose according to their own preferences, which can better meet the individual needs of the audience.

\subsubsection{Creating Traditional Sports Culture Brands with Yanzhao Characteristics}

Hebei Province has thousands of years of splendid culture, covering a very rich and diversified local cultural element and decorating colorful traditional cultural scenery. During the process of inter-cultural communication of Yanzhao traditional sports culture, it is necessary to choose representative sports instead of covering everything as much as possible and. Therefore, there are many choices for the development of modern sports culture brands. When creating traditional sports culture brands with rich Yanzhao characteristics, it is necessary to pay attention to the investigation and refinement of local cultural elements, so that the traditional sports culture of Yanzhao and the sports spirit of Yanzhao will live (Chen, 2017). Work hard to excavate the inheritance of Hebei's sports spirit, plan sports cultural boutique events and brand activities, and actively create highlights of demonstration. Sort out the traditional sports items in the list of intangible cultural heritage of Hebei Province, highlight the key points, and deepen the understanding and perception of creating traditional sports culture brands, so as to achieve cultural identity and innovation inheritance, and continue to develop innovative expression forms of traditional sports culture in the development of modern sports, incorporating fresh viewpoints and understandings in inter-cultural communication.

\subsection{Channels of Inter-Cultural Communication}

\subsubsection{Strengthening the Publicity of Yanzhao Traditional Sports Culture}

The publicity of sports culture promotion is to introduce, report, and publicize 
the cultural exchange activities of Yanzhao sports cultural traditions, consciousness, concepts, connotations, etc. by means of translating into foreign languages. This kind of activity not only has the general characteristics of language and text translation, which converts one language into another language. At the same time, it highlights the actual effect, audience's feedback, and the positive construction of the national image. Generally speaking, the sports culture will be translated into English, since it is a worldwide used language. Thus, it is convenient for people from other countries in the world to learn and understand Chinese sports culture, achieving the purpose of inter-cultural communication.

\subsubsection{Promoting the Digital Development of Yanzhao Traditional Sports Culture}

In the era of big data, high-tech and digital information should be used as soon as possible in all aspects of traditional sports culture management, especially in inter-cultural activities and services of traditional sports, to create an information database of Yanzhao traditional sports culture and to establish a comprehensive management system of traditional sports culture (Huang, 2008). It will be of great help to improve the management of data, to record, categorize and archive the outdated and fragmented data, and to preserve the complete traditional culture as much as possible. It is necessary to design and innovate an integrated inter-cultural communication model through the establishment of a comprehensive sports culture management model and the exploration of the connotation of local culture. For the inter-cultural communication of Yanzhao traditional sports culture, more innovations in modern management technology should be sought to provide more creative space for the development of sports in Hebei Province.

\subsubsection{Building a Strong Inter-Cultural Communication Platform for Yanzhao Traditional Sports Culture}

More attention should be paid to the construction of multiple communication channels for the inter-cultural communication of Yanzhao traditional sports culture. Modern three-dimensional communication platforms, diversified communication modes and careful design of local cultural elements should be adopted to stimulate sports passion, tell the story of Yanzhao sports and spread the voice of Yanzhao sports and the positive energy of Hebei sports culture. Diversified communication channels should be constructed, including official exchanges, independent exchanges of non-governmental organizations and commercial exchanges, which will strengthen physical exchanges of Yanzhao traditional sports culture in form of various subjects. A three-dimensional communication platform should be created that integrates traditional media and digital media. For example, exquisitely designed and graphic brochures of English edition and the English readings of Yanzhao traditional sports culture should be compiled to introduce systematically the origins of Yanzhao traditional sports culture. Digital media publicity works, English as the carrier, should be exploited for Yanzhao traditional sports culture. The method of illustration and combina- 
tion of viewing and listening will enhance the appeal, deepen the audience's understanding of sports culture, and let them appreciate the unique charm of Yanzhao traditional sports culture better.

\subsubsection{Integrating the Development of Sports Tourism Industry and Promoting Collaborative Communication}

The inter-cultural communication of Yanzhao traditional sports culture is not limited to participating in sports competitions. It also includes other rich models, such as sports tourism industry and sports cultural industries. Yanzhao traditional sports culture has obvious regional characteristics. Martial arts in Cangzhou, Yongnian Taichi in Handan, and fishing activities in Baiyang Lake all have their own characteristics. It can be combined with tourism projects to explore its industrial value, with the help of the natural beauty and cultural characteristics of various regions. Local historical customs and corresponding local traditional sports culture can be introduced to foreign tourists, which can break through the regional restrictions and make the public from all over the world feel the infinite charm of Yanzhao traditional sports and be influenced by its artistic influence. The degree of audience's participation in Yanzhao traditional sports culture tourism activities directly affects the effect of inter-cultural communication of Yanzhao traditional sports culture. The 2022 Beijing-Zhangjiakou Winter Olympics has created a new opportunity for it. The integration of the sports tourism industry with Yanzhao traditional sports culture can further develop sports culture in Hebei Province and attract a large number of tourists to Yanzhao traditional sports culture, promoting the new development of the sports culture industry.

\section{Conclusion}

The purpose, taking advantage of the opportunity of 2022 Winter Olympics, to carry out inter-cultural communication of Yanzhao traditional national sports is to output the excellent connotations and paradigms in the traditional sports culture of Yanzhao, so that Chinese people can deepen their sense of pride in traditional sports culture and build a strong province of sports culture, and deepen the target audience's understanding of Yanzhao traditional sports culture. To seize the opportunity of the Winter Olympics to carry out inter-cultural communication of Yanzhao sports culture, attention should firstly be paid to enhancing the awareness of the main body of inter-cultural communication, and the training of talents for inter-cultural communication. Secondly, the contents of inter-cultural communication should be carefully selected and build a three-dimensional system including national-level intangible cultural heritage-provincial intangible cultural heritage-municipal intangible cultural heritage. Last but not least, various channels should be built to intensify external publicity and increase international visibility through performances or international competitions. The combination of digital construction, characteristic communication platforms and sports tourism industry will definitely promote 
the inter-cultural communication of Yanzhao traditional sports culture.

In his report to the 19th National Congress of the Communist Party of China, General Secretary Xi Jinping emphasized that "We will improve our capacity for engaging in international communication so as to tell China's stories well, present a true, multi-dimensional, and panoramic view of China, and enhance our country's cultural soft power." Under the background of telling China's stories and spreading the voice of China, it is of great importance to take advantage of the opportunity of the 2022 "Beijing-Zhangjiakou Winter Olympics" to promote the spread of sports culture with Yanzhao characteristics such as Cangzhou martial arts, Handan Taichi, and Wuqiao Acrobatics. The story of Yanzhao traditional sports culture will surely become the paving stone for foreign tourists to understand the heavy Yanzhao culture, and even play a greater role as a link.

On account of various constraints, the present study has certain limitation. The strategies of inter-cultural communication of Yanzhao traditional sports are mainly discussed from theoretical perspective. Considering the actual situation of Hebei Province, how they can be put into practice in limited time is a great challenge. Further studies and exact measures should be taken as soon as possible so as to make full use of 2022 Winter Olympic Games and achieve the goal of promoting inter-cultural communication of Yanzhao traditional sports.

\section{Project}

This article is one of the results of the project of 2019 Hebei Province Social Science Development Research, Project Name: Research on the Inter-cultural Communication of Yanzhao Traditional Sports Culture under the Background of the Winter Olympics, Project Number: 2019030502009.

\section{Conflicts of Interest}

The authors declare no conflicts of interest regarding the publication of this paper.

\section{References}

Chen, G. (2017). Promotion of International Communication of Sports Culture in Implementation of the One Belt and One Road Strategy. Journal of Capital University of Physical Education and Sports, 29, 4-7+25.

Huang, Y. P. (2008). From "Hard Communication Times" to "Soft Communication Times": Inquiring about Historically "Hard Communication" Phenomenon Trend. Jilin Normal University Journal (Humanities \& Social Science Edition), 2, 34-38.

Jing, Q., Gai, H. H., \& Xu, J. C. (2010). The Feasibility Study about Heritage and Protection of Hebei Traditional Physical Culture in Colleges and Universities. Hebei Academic Journal, 30, 218-220.

Tuo, P. X. (2017). The Value, Dilemma and Dispelling of Cross-Cultural Communication of National Traditional Sports under the "All along the Way" Strategy. Journal of Sports Research, 31, 13-17. 